\title{
9ème Congrès biennal de la Société Asiatique et Océanique pour la Recherche sur la Sexualité et I'Impuissance (Asia Pacific Society for Sexual and Impotence Research)
}

\author{
Jacques BUVAT \\ CETPARP, Lille
}

Le Congrès biennal du chapitre asiatique et océanique de I'International Society for Sexual and Impotence Research s'est tenu à Cebu City, aux Philippines, du 1er au 4 Octobre 2003, réunissant 380 personnes, dont de nombreux orateurs invités du monde entier. J'ai sélectionné quelques présentations parmi les plus intéressantes:

Oestrogènes, phyto-oestrogènes, voire xéno oestrogènes peuvent-ils créer des problèmes d'érection ? (Oestrogen causes erectile dysfunction : A physiological crosstalk with androgen, abstract p. 77).

B. Srilatha a présenté des résultats récents du laboratoire du Pr Adaikan, de Singapour. Chez le rat, la " qualité » de l'érection, qu'on mesure par le paramètre augmentation de la pression intra-caverneuse en réponse à la stimulation électrique du nerf caverneux, se détériore avec l'âge, fournissant ainsi un modèle expérimental des problèmes d'érection liés à l'âge. L'étude rapportée par le Dr Srlilatha a montré que chez cet animal, une administration prolongée d'estradiol (12 semaines) accélérait cette détérioration de l'érection. L'administration d'estradiol est ainsi suivie d'une diminution significative des érections résultant de la stimulation du nerf caverneux. Les anomalies observées sont corrélées aux taux sériques d'estradiol (multipliés par 2 à 5 par rapport à la normale) et à la diminution de ceux de testostérone, elle-même secondaire à l'augmentation des taux d'estradiol qui exerce un rétrocontrôle négatif sur les centres gonadotropes hypothalamo-hypophysaires. Les études in vitro montrent que l'exposition à l'estradiol est responsable d'un défaut fonctionnel par déficit en monoxyde d'azote. On observe aussi des anomalies histologiques des corps caverneux. Les phyto-oestrogènes ont les mêmes effets. Reste à voir si les oestrogènes peuvent aussi perturber la fonction érectile dans l'espèce humaine. II faudrait alors évoquer que puisse aussi exister un effet toxique des xéno oestrogènes, c'est à dire des substances à activité oestrogénique qu'on trouve dans l'environnement, comme les pesticides dont une étude sud-américai- ne a récemment montré qu'ils semblaient, au moins en cas d'exposition massive, induire des troubles de l'érection chez certains hommes.

Les auteurs ont conclu leur présentation en soulignant l'intérêt potentiel, dans de tels cas de troubles de l'érection induits par les oestrogènes, des inhibiteurs de l'aromatase comme la testolactone et le levotrazole.

Le Losartan, un inhibiteur des récepteurs de l'angiotensine de type 1 améliore la fonction sexuelle des rats âgés (Erectile capacity of old rat can be enhanced by brief modulation of angiotensin receptor type 1, JaeSeung Paick et coll, abstract p. 10).

De nombreux autres travaux de recherche fondamentale ont été présentés au congrès de Cebu. Parmi ceux-ci une étude de plus en faveur du rôle du système rénine-angiotensine dans les problèmes d'érection liés à l'âge. L'angiotensine II est un vasoconstricteur puissant, sécrété dans les corps caverneux humains, où elle peut contracter le muscle lisse vasculaire et empêcher l'érection comme cela a été démontré chez le chien. Dans cette étude en double-insu contre placebo, un inhibiteur des récepteurs de l'angiotensine $1 \mathrm{I}$, le Losartan, a augmenté la réponse érectile des rats âgés à la stimulation électrique du nerf caverneux jusqu'aux valeurs des animaux jeunes, bien qu'il ait simultanément diminué la pression artérielle. Ce travail évoque donc la possibilité d'un intérêt thérapeutique des inhibiteurs des récepteurs de l'angiotensine II dans les problèmes d'érection liés à l'âge. Une étude publiée dans le numéro de Décembre 2003 de l'International Journal for

Correspondance :

jacques-buvat@wanadoo.fr 
Impotence Research (Hamed et al, I.J.I.R., 2003, $15: 418$ ) fournit en effet quelques arguments en faveur d'une implication du système rénine-angiotensine dans les problèmes d'érection de l'homme puisque les auteurs ont trouvé une augmentation significative des taux d'enzyme de conversion de l'angiotensine dans les corps caverneux de sujets présentant des problèmes d'érection (cet enzyme de conversion augmente les taux d'angiotensine II par conversion de l'angiotensine I). L'intérêt thérapeutique des inhibiteurs des récepteurs de l'angiotensine II reste cependant pour l'instant à confirmer dans notre espèce.

A propos de l'infidélité philippine (Philippine " non monogamy " : A multiplicity of relationships, abstract p. 20).

E.V. Macalalag et al. ont fait une enquête aux Philippines à propos de ce que Blumstein et Schwartz ont qualifié en 1983 de " non-monogamie " pour décrire l'activité sexuelle en dehors d'une relation de couple marié ou non, afin de dépasser la notion classique de sexualité extra-conjugale. Deux mille trois cent cinquante cinq personnes vivant en couple ont répondu à cette enquête, dont 1357 hommes et 998 femmes, âgés de 16 à 89 ans. Ces répondants ont été divisés en hétérosexuels, mariés ou non mariés, et homosexuels, hommes ou femmes. Cinquante deux pour cent des hommes et $14 \%$ des femmes hétérosexuels avaient des relations sexuelles hors couple. Les proportions étaient de $25 \%$ chez les homosexuels hommes ef $33 \%$ chez les lesbiennes. Parmi les hétérosexuels, c'était chez les sujets mariés que le taux d'infidélité était le plus élevé. Enfin c'est entre 30 et 60 ans que les relations sexuelles hors couple étaient les plus fréquentes.

Bien sûr à n'extrapoler qu'avec réserve aux populations non asiatiques !

Imagerie des centres nerveux de l'excitation sexuelle [Blood oxygenation level dependant (bold) functionnal magnetic resonance imaging for evaluating for cerebral centers of sexual arousal response, abstract p.88]

Les sud-coréens S. W. Kim et al. ont rapporté une nouvelle étude utilisant l'Imagerie en Résonance Magnétique Nucléaire fonctionnelle pour localiser les centres de l'excitation sexuelle dans l'espèce humaine. Comme cela a été le cas dans plusieurs études précédentes, c'est principalement au niveau du noyau caudé et du gyrus cingulaire qu'ils ont observé une activation spécifique à la suite de la projection de films érotiques, simultanément à la survenue d'une réponse sexuelle subjective évaluée par autoquestionnaire. La comparaison avec la réponse cérébrale à des films non érotiques avait permis d'identifier et de ne pas inclure dans ces zones " sexuelles » les zones activées par des stimuli non spécifiques (essentiellement les zones occipitales). L'application de cette méthode à l'exploration de situations pathologiques a débuté dans leur centre.

Impact sexuel de l'incontinence d'effort et de ses traitements (Impact of minimally invasive anti-incontinence therapy on the sexual life of women with stress urinary incontinence, abstract p. 27)
Les coréens $B$. Hong et al. ont évalué de façon prospective l'impact sexuel de l'incontinence urinaire d'effort et de ses traitements chez 22 femmes d'âge moyen 50 ans. Deux types de traitements ont été utilisés : soit un procédé chirurgical mini-invasif (bandelette vaginale type TVT), soit une rééducation de la musculature pelvienne par stimulation électrique externe. Soixante dix sept pour cent de ces femmes déclarèrent que leur problème urinaire avait perturbé de façon importante leur vie sexuelle. Sept des 10 femmes opérées, et 4 des 7 femmes traitées par rééducation, déclarèrent que le traitement de leur incontinence d'effort avait amélioré leur fonction sexuelle. Le score du Female Sexual Function Inventory (FSFI) augmenta significativement après pose du TVT, et dans la même proportion, quoique non significativement du fait du petit nombre de femmes traitées non chirurgicalement, dans le groupe rééduqué.

Cette étude tend à confirmer la fréquence de l'impact sexuel de l'incontinence d'effort, et suggère que son traitement peut, indirectement, améliorer la sexualité. Le faible nombre de femmes incluses limite cependant la portée de ces conclusions.

Raisons de l'arrêt du Sildénafil en dépit de la restauration d'une fonction érectile normale (Reasons for dicontinuation after successful restoration of erectile function with Sildenafil medication, abstract p.33).

Les coréens Son et al. ont étudié 156 hommes dont le score du domaine de la fonction érectile de l'IIEF s'était normalisé (score de 26 ou plus) sous traitement par le Sildénafil. Six mois après la première prescription de Sildénafil, ils ont évalué leur devenir au cours d'une consultation ou d'un entretien téléphonique. Un tiers $(34,6 \%)$ de ces 156 hommes qu'on aurait pu considérer comme des succès du traitement l'avait interrompu. Les raisons données étaient liées à la partenaire, particulièrement au fait que souvent elle n'était pas prête émotionnellement à reprendre une vie sexuelle après une période prolongée d'interruption (37\%), à la crainte d'effets négatifs sur leur santé $(18,5 \%)$, au retour d'une fonction érectile spontanément normale (15\%), à l'arrêt du traitement par Sildénafil du fait de traitements concomitants instaurés pour d'autres affections ( $11 \%)$, à la déception causée par l'absence de retour d'érections spontanées, le patient n'acceptant pas des érections ne pouvant être induites que par le médicament $(7,5 \%)$, au coût du traitement (4\%), à l'impossibilité de s'adapter à la programmation de l'activité sexuelle ( $4 \%)$ et au manque d'intérêt sexuel $(4 \%)$.

On voit donc qu'en dehors du petit groupe de patients guéris, et n'ayant plus besoin du traitement (de l'ordre de $5 \%$ de la population entière), la plupart des causes d'arrêt du traitement étaient liées à des problèmes psychologiques ou relationnels, ce qui confirme s'il en était besoin que rétablir une érection rigide ne suffit pas pour restaurer une vie sexuelle satisfaisante, pas plus chez les asiatiques que chez les européens. 